Correction

\title{
Correction: Zhu, Q., et al. Hyperspectral Remote Sensing of Phytoplankton Species Composition Based on Transfer Learning. Remote Sensing 2019, 11, 2001
}

\author{
Qing Zhu ${ }^{1}$, Fang Shen ${ }^{1,2, *} \mathbb{C}$, Pei Shang ${ }^{1}$, Yanqun Pan ${ }^{1}$ and Mengyu Li ${ }^{1}$
}

1 State Key Laboratory of Estuarine and Coastal Research, East China Normal University, Shanghai 200241, China; 52163904005@stu.ecnu.edu.cn (Q.Z.); 52142601008@stu.ecnu.edu.cn (P.S.);

52132601006@stu.ecnu.edu.cn (Y.P.); 52183904017@stu.ecnu.edu.cn (M.L.)

2 Institute of Eco-Chongming (IEC), East China Normal University, Shanghai 200062, China

* Correspondence: fshen@sklec.ecnu.edu.cn; Tel.: +86-021-5483-6003

The authors wish to make the following corrections to this paper [1]:

1. Figure 1 had 20 validation stations that were unlabeled, so the station labels were added.

Replace:

Figure 1 (Old Figure)

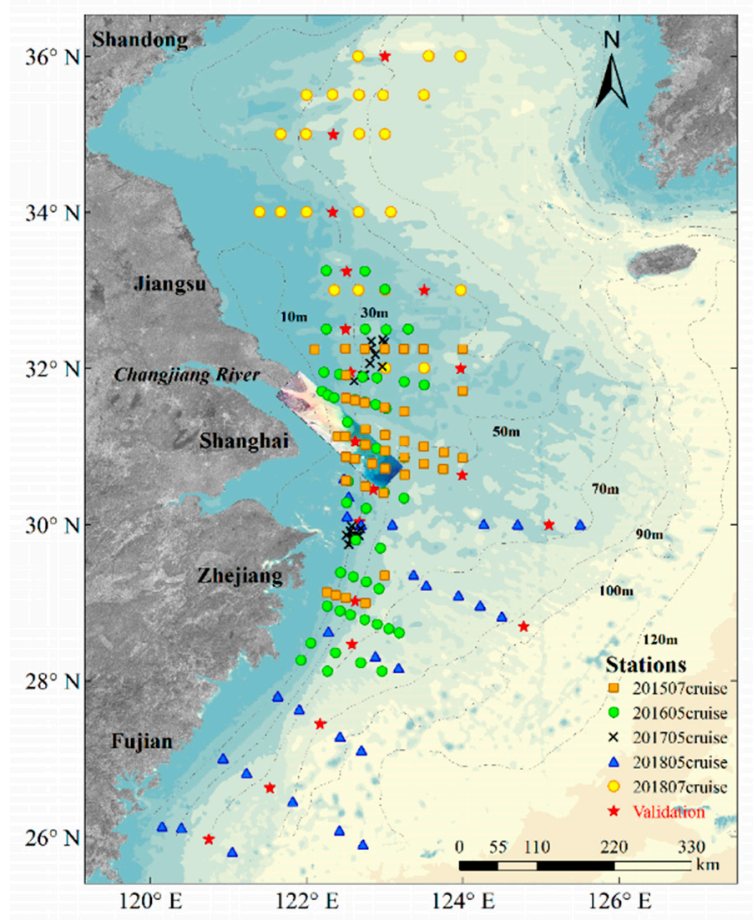

with 
Figure 1 (New Figure)

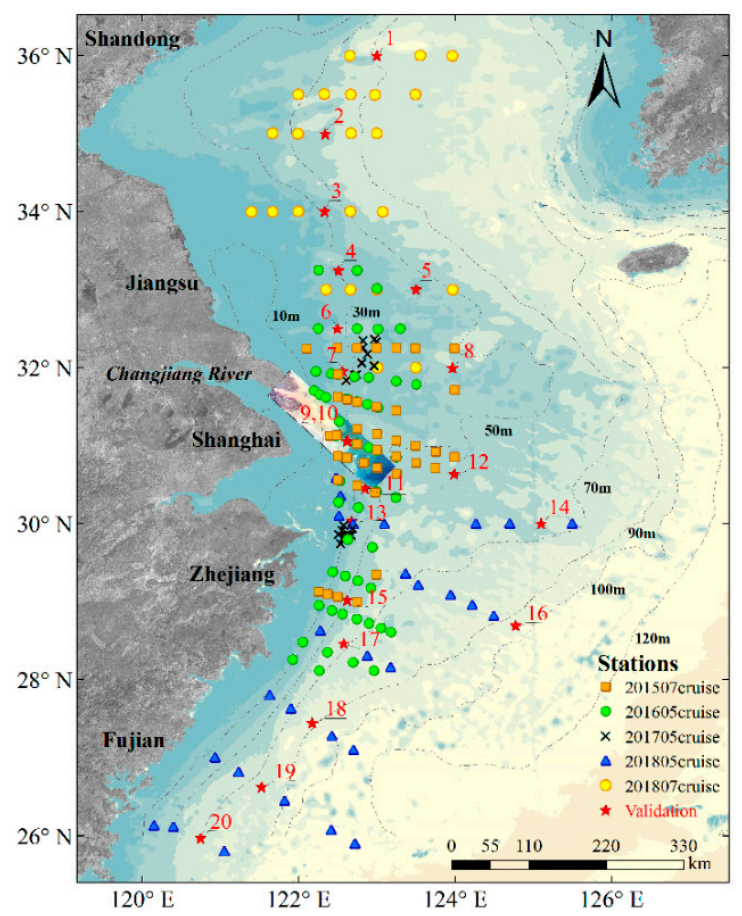

2. Figure 10 had a confusion in the number of stations (No. 4-15), and Figure 10 and Figure 11 had a reversal on the color plate of chrysophyta and chlorophyta (phytoplankton community) in the bar and the scatter plot, so these were corrected. Replace:

Figure 10 (Old Figure)

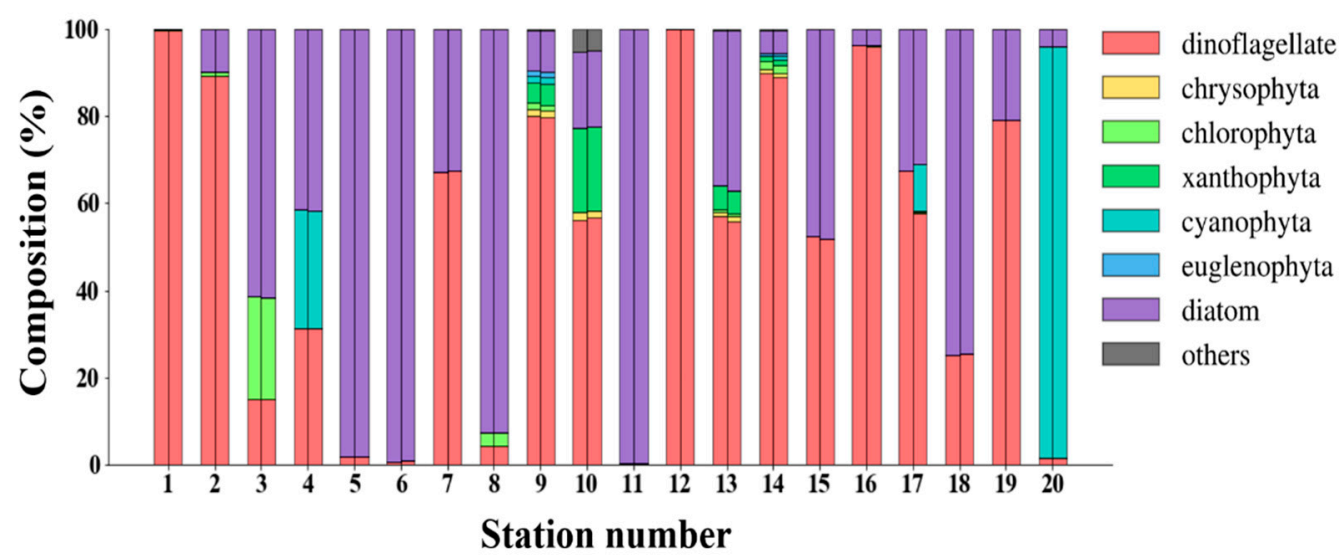

with 
Figure 10 (New Figure)

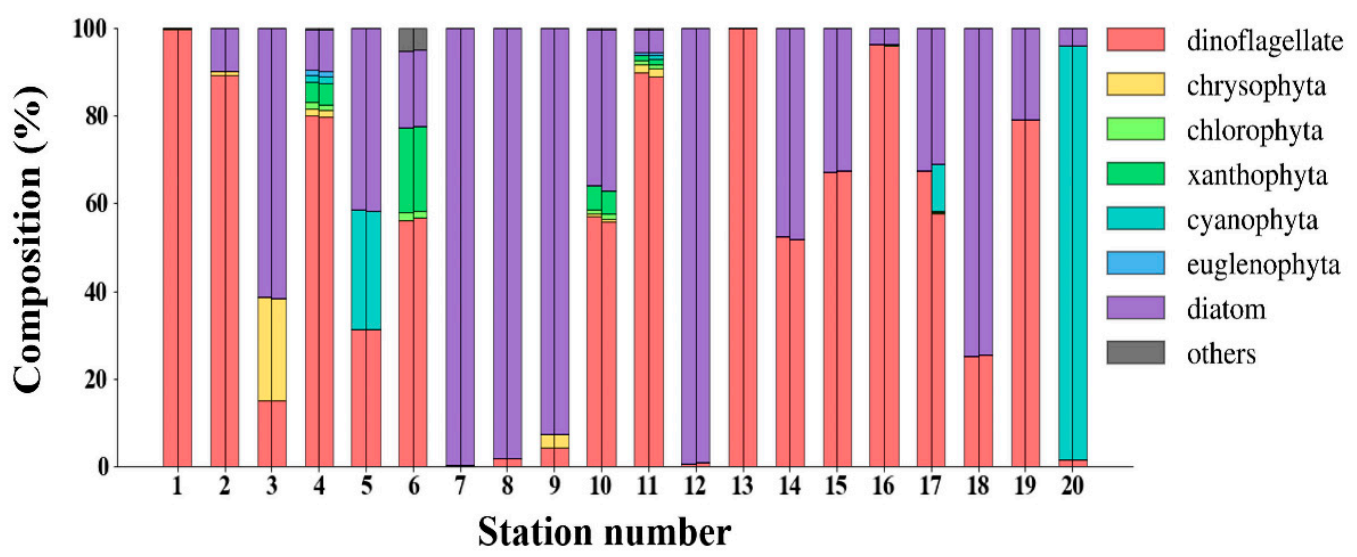

Replace:

Figure 11 (Old Figure)

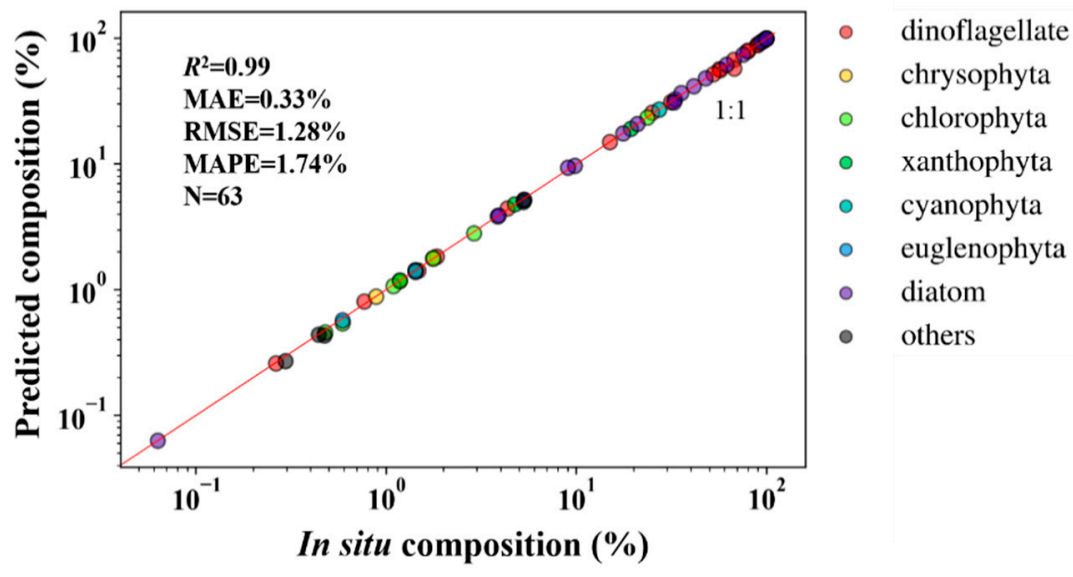

with

Figure 11 (New Figure)

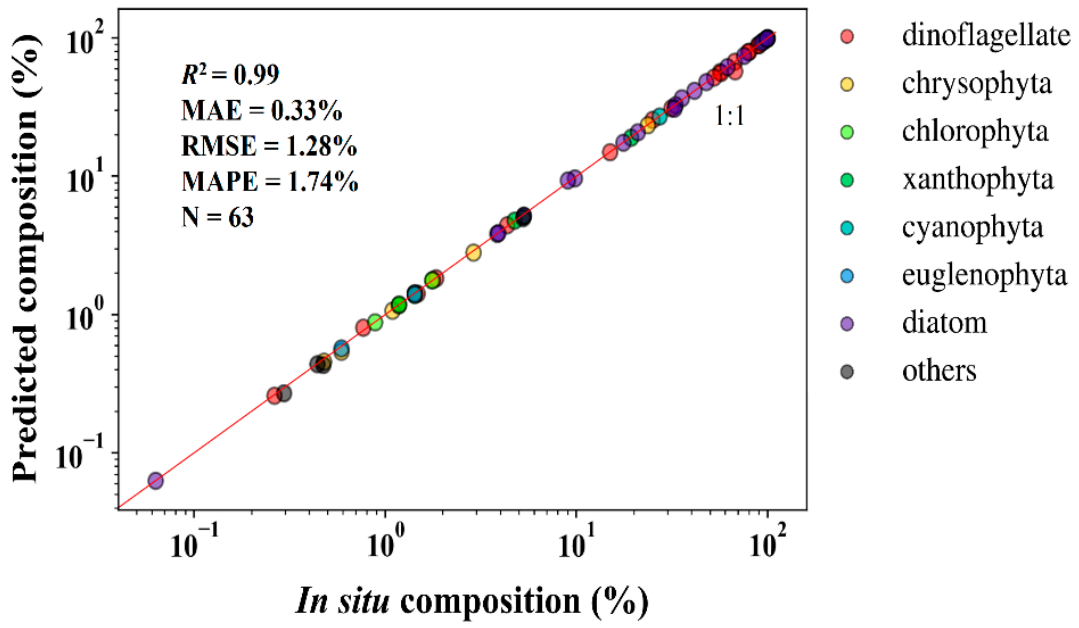


The authors confirm that these drawing mistakes have nothing to do with the science and technology of the published paper itself. The authors would like to apologize for any inconvenience caused to the readers by these changes.

\section{Reference}

1. Zhu, Q.; Shen, F.; Shang, P.; Pan, Y.; Li, M. Hyperspectral Remote Sensing of Phytoplankton Species Composition Based on Transfer Learning. Remote Sens. 2019, 11, 2001. [CrossRef] 